\title{
Chapter 6
}

\section{Pilocytic Astrocytoma: Anatomic, Pathological and Molecular Aspects}

\author{
Aline Paixao Becker, Cristovam Scapulatempo-Neto, \\ Luciano Neder, Leila Chimelli and Rui M. Reis
}

Additional information is available at the end of the chapter

http://dx.doi.org/10.5772/53956

\section{Introduction}

\subsection{Epidemiology}

Brain tumors are rare neoplasms, however, they represent the second commonest cancer in childhood (the first being hemato-lymphoid neoplasms) and correspond to the main solid tumor in the pediatric context (ages 0-19 years) [1]. In this setting, gliomas, and particularly astrocytomas are the leading group. Pilocytic astrocytoma (PA) is the commonest brain tumor in the ages 5-14 years and the second in the age ranges 0-4 years and 15-19 years, although it represents about only $6 \%$ of all gliomas, according to the last Central Brain Tumors Registry (CBTRUS) Report [1]. PA affects males and females equally, and the main affected ages are 6 to 13 years-old, with $75 \%$ of cases occurring at this ages [2; 3].

PAs present a good prognosis in general, with 10-year survival $>90 \%$ [4]. However, about $10-20 \%$ of patients suffer with recurrence of completely excised lesions or growing of residual lesions [4]. Besides, 2-3\% of the cases may disseminate through the spinal cord [2;4]. These aggressive tumors are particularly more frequent in adults and older patients, which explains the difference in the 10-year survival [5].Overall, the prognosis between children and adults is significantly different [5]. While in children and young adults the 5 -year survival is $>90 \%$, in the group of $60+$ years patients, this value is about $52 \%$ [5]. Likewise, the mortality rates related to PAs are higher in adults than in children. Despite this could be explained by differences in the location of these tumors in adults many evidences suggest that PA is in fact a more aggressive neoplasm in older age groups [5]. 


\section{Anatomical location and neurorradiological features}

The main location of PA is the cerebellum, with more than $60 \%$ of the cases occurring in cerebellar hemispheres, however, it can arise all through the neuraxis, including hypothalamic region, the optic-chiasmatic tract and spinal cord, with some cases involving the whole spinal cord ("holocord pilocytic astrocytoma") [2; 3; 6]- [9]. This wide distribution in the central nervous system (CNS) may, in part, explain the differences in the prognosis of PA, since GTR of a lesion located near or in eloquent areas of the CNS may be unachievable. In addition, some locations present special features or are related to specific conditions. For example, multiple tumors and tumors located in the optic-chiasmatic region are far more common in patients with neurofibromatosis 1 (NF1), while supratentorial and spinal cord PAs are more frequent in adults than in children $[2 ; 5]$.

At neurorradiological exams, the typical finding is, generally, a non-infiltrative, well delimited biphasic lesion, with a cystic, hipointense, area associated with a mural nodule, which can be contrast-enhanced at magnetic resonance image (MRI) and computed tomography (CT), however, diverse patterns as solid nodule or complete cystic lesions can be seen [2;10] (Figure 1). In any case, perilesional edema is not commonly observed, as it is in high-grades primary brain tumors [11]. Anecdotally, PA can be manifested as cerebral hemorrhage [12].
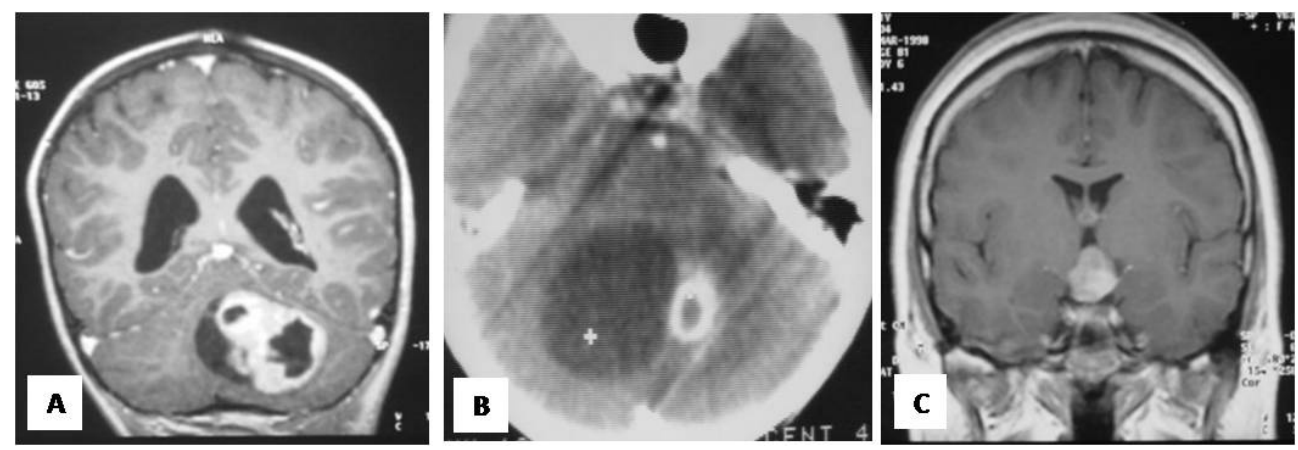

Figure 1. Neurorradiological features of Pilocytic Astrocytomas, after endovenous contrast injection. Note the absence of perilesional edema, even in voluminous lesions. A- Cerebellar lesion - solid with cystic areas. This is the commonest pattern of PA (MRI, coronal view). B- Cerebellar lesion, predominantly cystic with a mural nodule (CT, axial view). Csolid, suprasselar lesion (MRI, coronal view). 


\section{Clinical signs and symptoms}

Due to its highly variable location in the neuraxis, the signs and symptoms of PA depend on the affected area. As the most common location is the cerebellum, headache and neck pain, vomiting, gait disturbance and visual abnormalities are usually referred $[2 ; 3 ; 6 ; 13]$. In supratentorial tumors, the occurrence of seizures may be seen and is related to cortical involvement [6]. In the hypothalamic area, PA may present with hormonal dysfunctions, for example, diabetes insipidus, obesity [3; 11] and the diencephalic syndrome - emaciation, hiperkinesis, irritability and accelerated growth [11]. The involvement of the optical pathway is related to visual loss or visual-field deficits [6; 11]; proptosis presents only in large, intraorbital tumors [6].

Clinical signs of intracranial hypertension, as hydrocephalus, papilledema, nistagmus, nausea and vomiting are common in virtually all locations, because of the mass effect impinged by the neoplasm $[2 ; 3 ; 11]$. On the other hand, smaller lesions may remain asymptomatic by some variable time [11].

\section{Histopathological aspects}

Some histopathological features of PA are well-established, for instance, biphasic pattern, with high- and low-cellularity areas; elongated (piloid) and rounded, oligodendroglial-like, cells mixed in various proportions; microcystic areas; Rosenthal fibers, and eosinophilic granular bodies are the commonest findings [2; 6] (Figure 2). In general, these findings support a diagnosis of a low-grade astrocytoma (grade I), according to the World Health Organization (WHO) criteria. However, features like microvascular proliferation, necrosis and degenerative nuclear atypia, more commonly present in infiltrative, high-grade gliomas, may be seen (Figure 3B, C). Diverse from infiltrative tumors, though, these features shall not be interpreted as malignancy signs, unless they are associated to high mitotic activity [2; 3; 10]. Leptomeningeal dissemination is quite common, and apparently is not related to aggressive behavior [14]. Calcification, hemorrhage and perivascular lymphocytic infiltration are less common (Figure 3A, D), but may be seen in some cases [10; 14]. Eventually, neuronal cells can be observed, intermingled in the tumor, but they lack neoplastic features, and can be interpreted as entrapped neurons $[6 ; 10 ; 14]$.

Because of the great variation in histopathological appearance, the diagnosis of PA is usually challenging, especially in minute biopsies, and greatly dependent upon correlation with neurorradiological aspects [2; 3]. This can be such a dilemma, that some authors refer huge discordance between pathologists, with half of PAs misdiagnosed as higher-grade tumors at primary diagnosis [15]. 

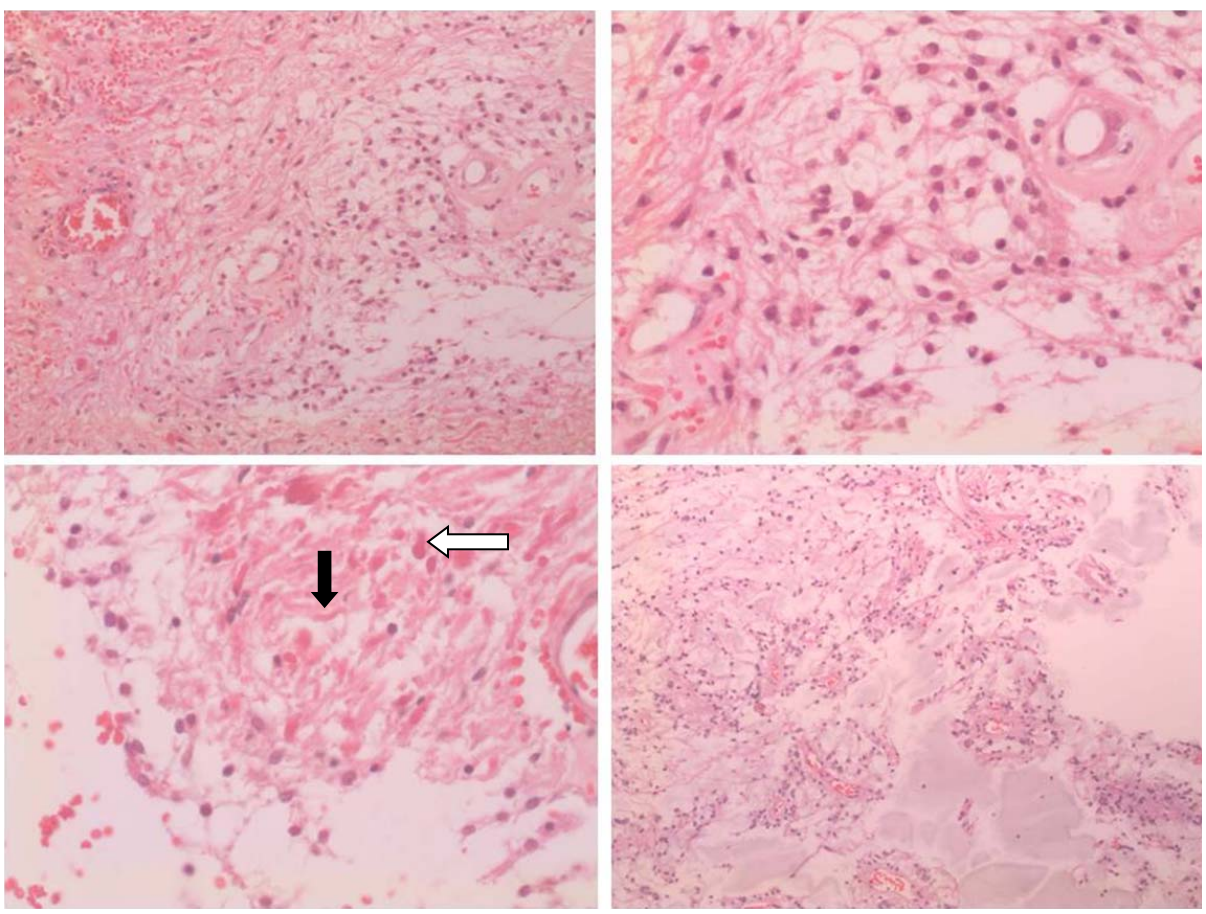

Figure 2. Histopathological aspects of Pilocytic Astrocytoma. All microphotographs represent H\&E stain. A- Biphasic pattern - high cellularity at left and lower cellularity at bottom right (100x). B- Round and piloid cells constitute this tumor. Note the vasculature, with typical endothelia (200x). C- Rosenthal fibers (black arrow) and eosinophilic granular body (white arrow) can be seen in higher cellular areas (200x). D- At bottom, a microcystic area is seen (40x). 

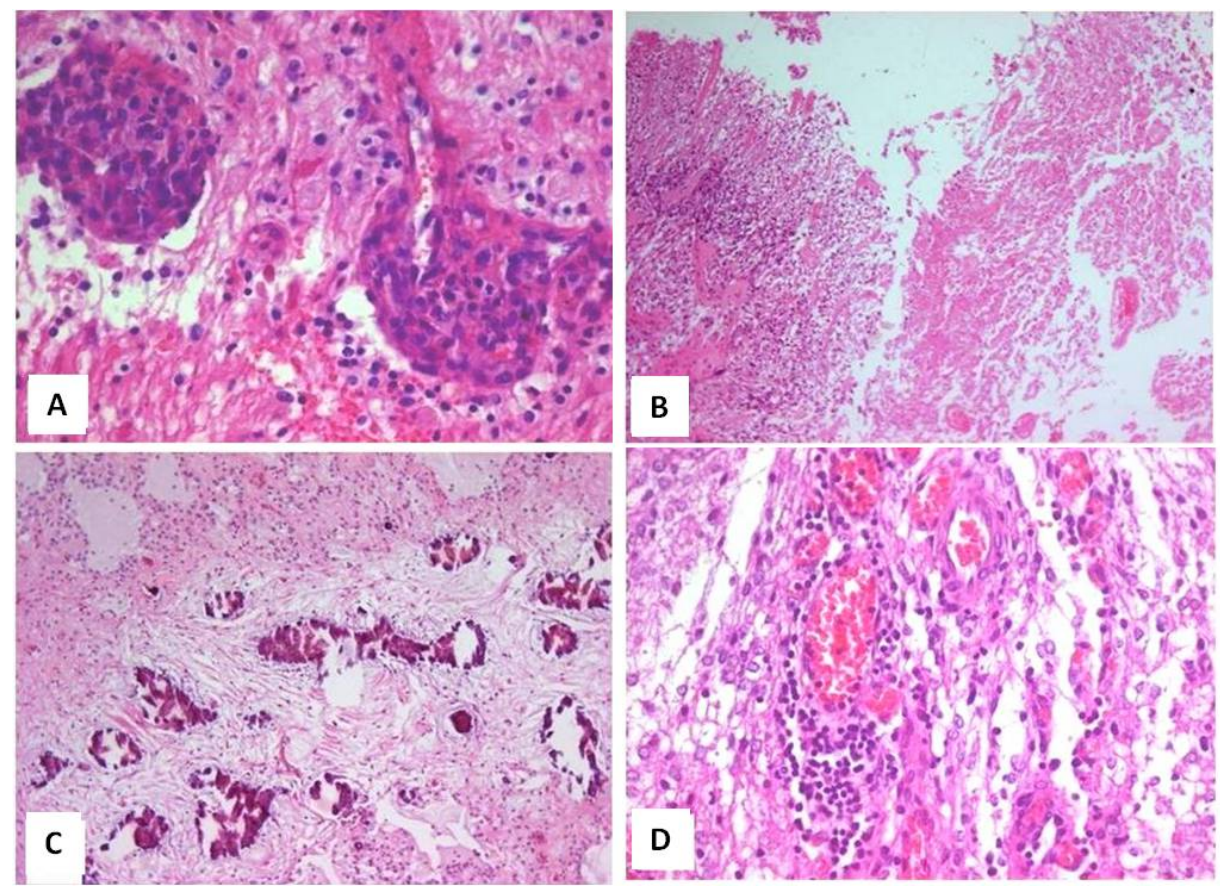

Figure 3. Some other histopathological aspects of Pilocytic Astrocytoma at the H\&E stain. A- Microvascular proliferation (200x) B-Areas of necrosis may be seen in some tumors - in this case, the necrosis is at right (40x). C-Calcification foci near a microcystic area of tumor (40x). D- Lymphocytic cuffs may be present (200x).

\subsection{Differential diagnosis}

Differential diagnoses of PA include low- and high-grade gliomas and the identification of different entities has great importance in the treatment choice and prognosis of patients.

Among low-grade tumors, the most important differential diagnoses include ganglioglioma; pleomorphic xantoastrocytoma (PXA); grade II oligodendroglioma; and diffuse astrocitoma. In these cases, tumor location, neurorradiological and immunohistochemical features can help in the definition, and this will be better discussed later in next sections. Moreover, hypothalamic lesions, especially in infants, must also be distinguished from pilomixoid astrocytoma, a recently described tumor, stated as grade II variant of PA in the last WHO classification $[2 ; 3]$.

On the other hand, anaplastic astrocytoma and anaplastic oligodendroglioma (WHO grade III) are some of the high grade lesions that can be confounded with PA [2;3] when there are degenerative atypia and/or oligodendroglial-like areas in the tumor. Finally, glioblastoma (GBM - WHO grade IV), the commonest and most aggressive astrocytoma, share various histological and neurorradiological features with PA, which makes the diagnosis problematic in some cases [16]- [18]. In these cases, clinical correlation and immunohistochemical markers can be used for better defining the diagnosis. 


\section{Immunohistochemistry in the diagnosis of pylocitic astrocytoma}

In order to reduce misdiagnoses, some immunohistochemical markers can be used to elucidate complicated cases. There are no specific markers for PA, but differences between expressions of standard antibodies can help in the task. Some of the main immunohistochemical markers are described herein:

\subsection{Glial Fibrillary Acidic Protein (GFAP)}

GFAP is a cytoplasmatic intermediate filament, component of the cytoskeleton of normal and neoplastic astrocytes [3]. Like in all astrocytomas, GFAP is persistently, diffusely expressed in PAs, which does not permit the delineation between PA and diffuse astrocytomas. However, it may be a useful tool to differentiate PA from other gliomas, such as ependymomas and oligodendrogliomas.

As described earlier, some PAs may present perivascular lymphocytic cuffs and neuronal cells entrapped among the neoplastic astrocytes. These characteristics are also seen in gangliogliomas [6]. To rule out this possibility, GFAP may help, as stated since 1970s by Eng and Rubinstein [19], since the neoplastic neuronal cells in this tumor are negative and may be highlighted in contrast to the strongly positive neoplastic astrocytes with this marker - this pattern corroborates the mixed nature of ganglioglioma. There are other immunohistochemical markers that help in this differential diagnosis, in addition to GFAP, for example, CD34 (positive in gangliogliomas and negative in PAs) and Neu N (neuronal marker, useful in the identification of neoplastic and entrapped neurons).

Secondly, since PAs may present oligodendroglial-like areas and calcifications, another utility for the use of GFAP is to differentiate these areas (in a small sample, for example) from oligodendrogliomas. Oligodendroglial tumors are mostly negative, however, a peculiar pattern of perinuclear immunoreactivity was recognized after the description of the protoplasmatic oligodendrocytes, present in almost $80 \%$ of oligodendrogliomas $[17 ; 20]$. Even in those positive cases, the pattern contrasts to the cytoplasmatic, diffuse positivity seen in the PAs. Other immunohistochemical markers can be used in a panel in order to refine this diagnosis, for instance, OLIG-2, a transcription factor involved in oligodendroglial differentiation [21]. Although some weak immunnopositivity may be seen in astrocytomas (low and high grades) and more than 70\% of PAs may be immunoreactive for OLIG-2 [22], according to some authors, OLIG-2 can significantly distinguish oligodendroglial and astrocytic tumors [20; 21]. As these both markers (GFAP and OLIG-2) can be simultaneously expressed by PAs and oligodendrogliomas, the interpretation of these immunohistochemical tests must take in account other histopathological findings and even neurorradiological features.

Finally, although GBM is a also a tumor of astrocytic lineage, it may present a different pattern of GFAP immunopositivity. Opposed to the diffuse positivity in PA, GBM is heterogeneously immunoreactive, predominant at the periphery of the tumor or only focal. This happens because the immunoexpression of GFAP tends to decrease with the malignant progression of astrocytomas $[16 ; 17 ; 23]$. 
In summary, GFAP is an important marker of astrocytic differentiation and can help to distinguish PA from other low- and high grade gliomas, both through the presence or absence of its immunoexpression and the different patterns (subcellular location and distribution over the tumor areas) of immunopositivity.

\section{2. $\mathrm{Ki} 67$}

The non-histone nuclear protein ki67 is a protein codified by genes present in the chromosome 10 [24; 25]. It is expressed in the nuclei of cells in all but G0 and early G1 phases of the cellular cycle [26], which allows a faithful estimative of the proliferative index of a neoplasm. As PA is a grade I astrocytoma, low proliferative index is the rule in these tumors [2; 3] (Figure 4A) with medium values about 2\% [14; 27], however medium values as high as $4,4 \%$ have been related [10; 28$]$.
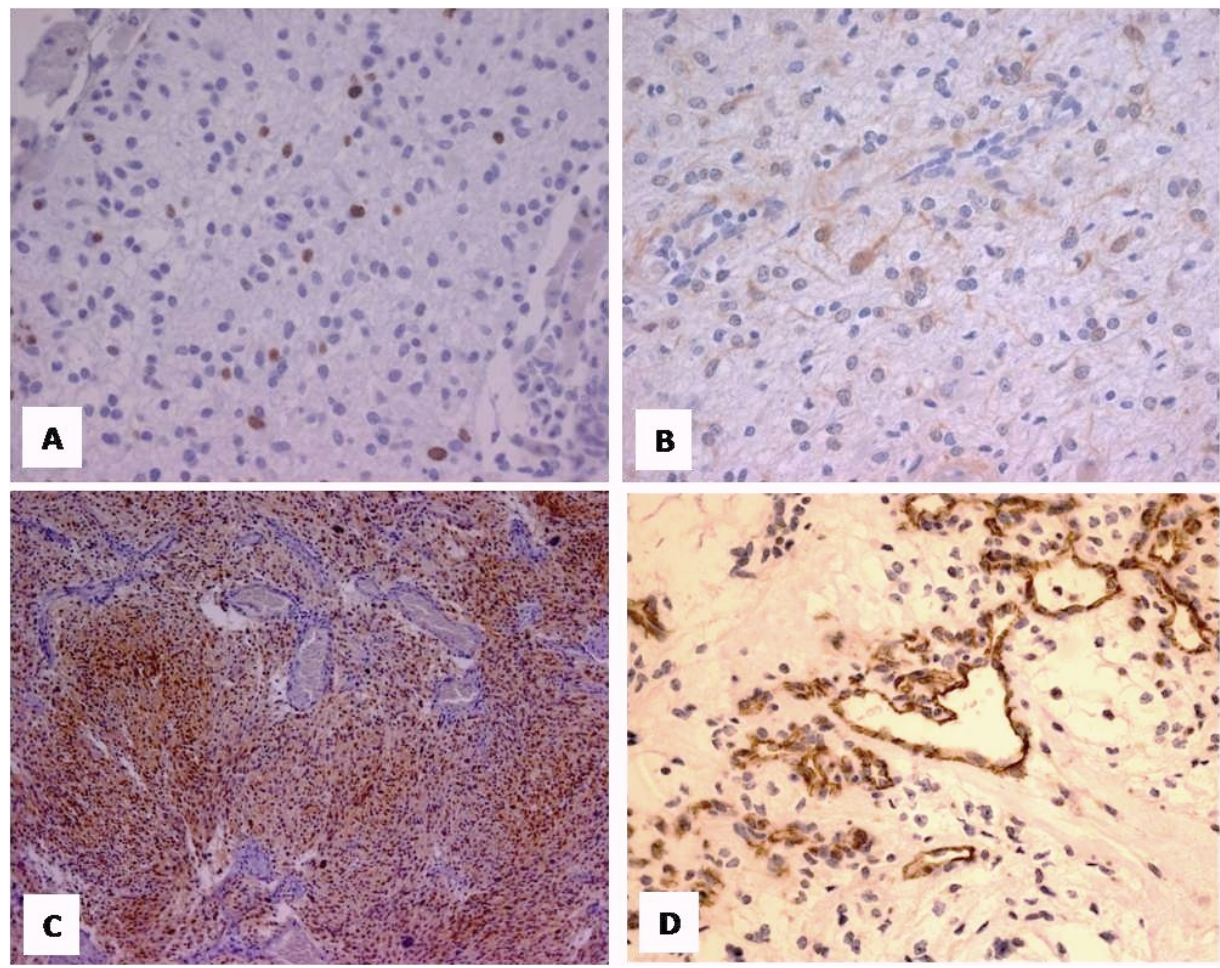

Figure 4. Immunnohistochemical aspects, with avidin-biotin-peroxidade technique. A- Ki67 - nuclear positivity in about $2 \%$ of neoplastic cells(100x). B- Galectin-3 - this case showed weak, diffuse immunopositivity and predominant cytoplasmatic location (100x). C- Galectin-3 strong, diffuse positivity and the vascular endothelium showed no reaction (100x). D- CD31 reaction in the endothelia - delicate vessels in the tumor (200x). 
Proliferative index by ki67 expression is a well established prognostic factor in diffuse astrocytomas [29], yet, its role in the prognosis of PA is controversial. In 2002, Roessler et al did not show significant differences between cerebellar PA when comparing groups according to the proliferative index with a cut-off value of $5 \%$ [28] and this result was further corroborated by other study with even lower cut-off value of 3\% [10]. Shortly after, Bowers et al (2003) described a subset of PA with worse prognosis, when the proliferative index determined by Ki67 was $>2 \%$ [27]. More recently higher medium values $(>10 \%)$ were described in recurrent and more aggressive PAs [10], and this exceptionally high value is similar to the medium value of proliferative index for GBM $(12,28 \%)$ described earlier by the same group [29].

Although the proliferative index is still a questionable independent prognostic factor of PAs, it is well accepted that a strict follow up is needed in the cases with high proliferative activity [10; 27].

\subsection{Galectin-3}

Galectin-3 (gal-3) is a carbohydrate-binding protein that binds specifically to $\beta$-galactosides sugars. This protein is involved in various biological processes, such as cellular proliferation, apoptosis, transcriptional regulation, intracellular signalization, adhesion and migration [30; 31]. The expression of gal-3 may be seen in both cytoplasm and nuclei of neoplastic cells (particularly carcinomas) from various organs, such as thyroid, pancreas and prostate [32], but the relation between hyper- or loss of expression and biologic behavior of the tumor varies among the different organs [32].

In APs, gal-3 is expressed in the nuclei and cytoplasm of neoplastic cells in all cases of PA described by various authors $[2 ; 10 ; 32 ; 33]$, but lacks the expression in the vascular endothelia(Figure 4B, C), contrary to what it is seen in non-neoplastic brain parenchyma [10; 33] and in astrocytomas grades II and III and oligodendroglial tumors, which permits a faithful delineation from PA when facing tough cases [17; 31].

Gal-3 is not specific of PA, since other low-grade tumors, such as ependymomas and PXA also present diffuse expression of gal-3 [10;32; 33]. Interestingly, focal (heterogenous) expression of gal-3 is also seen in GBM [2; 18;32], and although the vast differences between the biological behavior and molecular pathways of these tumors, this expression may be related to microvascular proliferation and breaking of hematological barrier (and contrast-enhancement at neuroimage) seen in these both entities $[10 ; 18]$.

Although various studies have tried to relate the intensity and extension of gal-3 expression to the clinical behavior of PA, no prognostic significance was proved until now. Still, as gal-3 expression is a conspicuous finding in PAs, in a pattern that can be differentiated from other grades of astrocytoma, this marker may be very useful in the tumor diagnosis $[10 ; 18 ; 32 ; 33]$. Besides, recently a group of authors described a close relationship between expression of gal-3 and activation of the RAF-MEK-ERK pathway in pancreatic cancer [34] and, as we shall see later in this chapter, that is an important molecular pathway in the genesis of PA, so gal-3 may be soon proved as a potential target for future treatments. 


\section{Molecular biology of pilocytic astrocytomas}

Since no prognostic factors have been identified to differentiate regular from the more aggressive Pas $[5 ; 10 ; 14]$, the genetic characteristics of the PAs have recently been investigated to better understand and try to predict the behavior of this tumor. Among these alterations, chromossomal abnormalities (structural or numerical, as the aneuploidies), single-gene mutations and epigenetic damage are mechanisms that could launch the molecular pathway of oncogenesis.

The molecular pathways of oncogenesis differ in PAs and diffuse astrocytomas. For example, two growth factor receptors, EGFR and PDGFR, related to invasion and malignant progression, which are frequently hyperexpressed in infiltrative diffuse astrocytomas had a lower expression in PAs observed by different groups [35;36]. Besides, other genes characteristically altered in diffuse gliomas, as TP53 and PTEN are normally expressed in Pas $[2 ; 35 ; 37]$.

No cytogenetic abnormalities were detected in PA at first, and the majority of PAs presented normal karyotype, similar to fetal astrocytes [37], and the majority of the altered tumors were from female and adult patients [2]. Since then, a lot of genes have been investigated. We shall see the most studied genes in PAs in the subsequent sessions.

\subsection{NF1 gene}

The first genetic studies tried to establish some differences between sporadic PAs and NF1PA, which present a more aggressive behaviour. It was known that NF1-PAs present germline mutations of NF1 (in contrast to somatic mutations in GBM of this gene) and this results in a loss of expression of the gene and, at protein level, results in a defective protein, neurofibromin [3], which lastly permits a constitutive activation of the RAS-RAF-MERK-ERK molecular pathway [4], or the mTOR/AKT pathway, activated in more aggressive Pas [4].

The detection of NF1 mutations can be achieved by molecular methods, by verifying the expression of the gene or even at IHC, through the expression (or loss of expression) of neurofibromin. $[2 ; 3]$

\subsection{The RAS-RAF-MEK-ERK signaling pathway}

The studies of NF1 gene were "the initial indication that Mitogen-activated protein kinase (MAPK) signaling might play a role in the development of PAs", as stated by Jones et al (2011) [4]. Since then, various groups have investigated the participant proteins of these pathways.

The MAPK pathways regulate fundamental biologic processes. In mammalian, there are 4 well-described MAPK pathways [38; 39] (Figure 5) and the most studied is the extracellular signal-regulated kinase (ERK)1/2 [39]. Alterations of this pathway are described in both low and high-grade astrocytomas [4], but molecular mechanisms differ between them.

In summary, the ERK1/2 pathway is triggered by the binding of soluble peptides to a Tyrosin-kinase receptor (TKR) at the cell surface, and initiates a cascade of events that terminates in the appropriated cellular response [40]. This pathway is related to various 


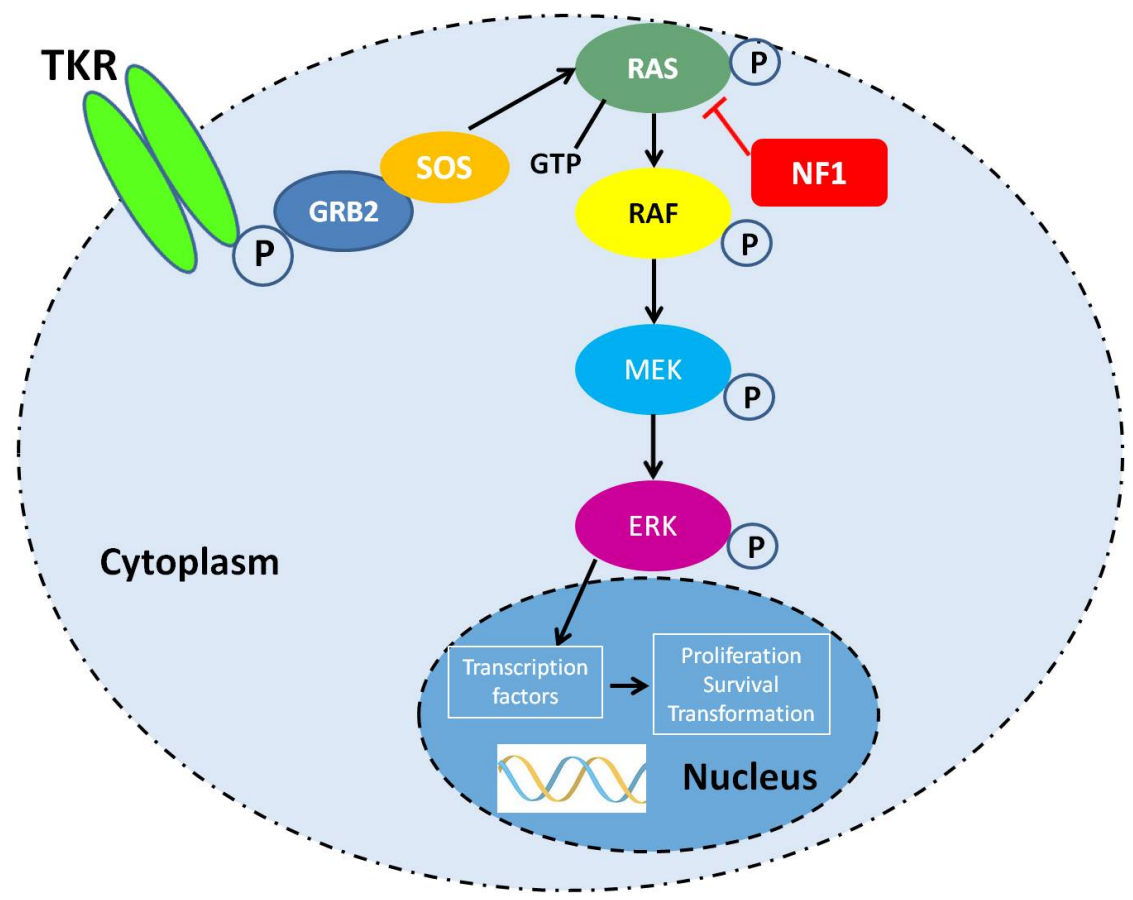

Figure 5. Integration of the MAPK pathways in the cellular response to various environmental stimuli. The sequential phosphorilation of RAS-RAF-MEK ERK, after dimerization of TKR, and the role of negative regulation of NF1.

fundamental cellular processes, such as proliferation, cellular differentiation, survival, migration, and angiogenesis [41; 42].

The ERK cascade is constituted by RAS, Raf, MEK and ERK proteins [39], which are subsequently activated during the process. After the binding of the extracellular peptide with the TKR and its dimerization, it activates the RAS protein, which then activates the Raf family, MEK and, finally, ERK protein [40; 43], that is present in the nuclei, to effectuate the response to the.initiator sign [43]. The RAS-Raf league is considered the regulatory center of the ERK cascade and mutations of any of this genes result in ERK pathway misregulation. It is important to note that the mutations of these proteins are mutually exclusive [39].

The proteins of Raf family are the main effectors of RAS and their action is in phosphorylating MEK 1 and MEK2 [44]. This family is a cytoplasmatic group of proteins, constituted by A-Raf, B-Raf and C-Raf [43], proteins that present similar structure and biological features [44], although they show different tissue distribution and capacity of activating the MEK proteins [45].

\subsubsection{Oncogene BRAF}

In humans, the most studied Raf protein is the B-Raf (BRAF), which is mutated in about $8 \%$ of all human neoplasms [44], and in melanomas this rate can be as high as $60 \%$ of the cases, with 
the commonest mutation being point mutation V600E [46; 47].In the setting of PA, mutations with constitutive activation of BRAF were demonstrated in up to $80 \%$ of cases, while they are very rare in diffuse astrocytomas [48]. The mechanisms that explain a constitutive activation of the BRAF are: i) point mutation V600E [44; 49; 50]; ii) duplication of a region in chromosome 7q [51; 52]; and iii) fusion between oncogenes BRAF and KIAA1549.

The point mutation V600E refers to a single glutamic acid for valin substitution at codon 600 of BRAF gene [44], which is the commonest BRAF missense mutation in human cancer [44; 46]. This mutation is not specific for PA and can be detected in various brain tumors, including PXAs, gangliogliomas, and in up to $6 \%$ of GBMs $[49 ; 50]$ and other tumors, such as thyroid cancers and melanomas [44].

The BRAF duplications are due to a somatic rearrangement of the gene in sporadic PAs. It was the first described genetic alteration of PAs [51;52]. They are more frequent in gliomas in noncerebellar [52] location, including that of the optical pathways [53], both in NF1-related tumors and in those sporadic ones. Nevertheless, other authors did not identify BRAF duplications in NF1-related tumors, and suggested the activation of a different pathway (mTOR) in the genesis of these frequently more aggressive tumors [54]

Finally, the fusion BRAF-KIAA1549 is present in $50-100 \%$ of the patients with PA $[4 ; 55 ; 56]$. This alteration is highly specific for PAs, and there is a growing tendency for using this marker as a powerful tool of molecular diagnosis in surgical pathology routine [57]. Although this fusion has recently been described in some other low-grade tumors, such as pilomixoid astrocytoma, glioneuronal tumors and unclassifiable low grade gliomas [57; 58], clinical and pathological correlation permit the diagnosis of PA in cases where diffuse component is predominant at histology [57]. Also, this fusion is significantly more frequent in infratentorial and optical pathways-located tumors [57].

The importance of studying the genetic mutations in PAs has grown, since many target therapies against the MAPKs pathway components are recently being developed. For example, concerning tumors with the V600E mutation, in 2012 two groups described the use of Vemurafenib, a BRAF inhibitor, in melanoma [59] and in lung cancer [60], with good results. In addition, other proteins of the ERK cascade can be targeted by novel drugs, as Trametinib, a specific MEK inhibitor, that has also been used successfully in patients with melanomas that present V600E mutation [61]. It is still unknown whether tumors harboring the fusion BRAF-KIAA1549 are responsive to Vemurafenib and Trametinib. Concerning the former, the distinct studies showed the lack of patient's response in the wildtype BRAF [62]

At last, it is important to note that the resultant protein of the fusion BRAF-KIAA1549 is cancer-specific and it is potentially a target for future treatments. The most representative example of this possibility is the identification of the fusion $B C R-A B L$ in chronic myeloid leukemia (CML), in a similar molecular mechanism. The knowledge of this fusion permitted the development of a target-drug, Imatinib, in the middle 2000's [63].This drug has changed the treatment and prognosis of the CML patients by controlling the disease, with excellent tolerability. 
The molecular pathways of PAs are beginning to be faithfully recognized and some of the molecules of these pathways have already some kind of target drug available for inhibition, with efficacy proved in other kinds of tumors. As there are no studies with these novel drugs in PAs, this can become a good direction for future clinical research.

\section{Conclusion}

Pilocytic astrocytoma is usually a tumor with good prognosis; however some eloquent locations do not permit the total resection of lesions. In these cases and in the ones that can recur even after gross total resection, the patients can suffer with physical limitations and even death. The study of the molecular pathways of PA's oncogenesis may represent a hope for longer and better quality life for these patients. There is a wide field for research in order to better understand this intriguing tumor, since the understanding of these mechanisms can turn a potentially fatal in a controllable disease.

\section{Acknowledgements}

The authors appreciatively thank Dr. Ricardo Santos de Oliveira, M.D., PhD and Dr. Hélio Rubens Machado, M.D., PhD (Department of Neurosurgery- FMRP-USP) for the neurorradiological exams photographs and the Surgical Pathology Department of FMRP-USP (SERPAT) for the microphotographs of the cases.

\section{Author details}

Aline Paixao Becker ${ }^{1,2}$, Cristovam Scapulatempo-Neto ${ }^{1,3}$, Luciano Neder ${ }^{4}$, Leila Chimelli ${ }^{5}$ and Rui M. Reis ${ }^{1,6}$

1 Molecular Oncology Research Center, Barretos Cancer Hospital, Barretos, São Paulo, Brazil

2 School of Medicine, University of Ribeirão Preto (UNAERP), Brazil

3 Department of Pathology, Barretos Cancer Hospital, Barretos, São Paulo, Brazil

4 Ribeirão Preto School of Medicine, University of São Paulo (FMRP-USP), Brazil

5 Division of Pathology National Institute of Cancer, Brazil

6 Life and Health Sciences Research Institute (ICVS), Health Sciences School, University of Minho, Braga, Portugal 


\section{References}

[1] CBTRUS Statistical Report: Primary Brain and Central Nervous System Tumors Diagnosed in the United States in (2012). Ref Type: Report

[2] Scheithauer, B. W, Hawkins, C, \& Tihan, T. VandenBerg SR, Burger PC. Pilocytic astrocytoma. In: David N.Louis, Hiroko Ohgaki, Otmar D.Wiestler, eds. WHO Classification of tumours of the central nervous system. Lyon: IARC, (2007). , 2007, 14-21.

[3] Louis, D. N, Reifenberger, G, Brat, D. J, \& Ellison, D. W. Tumours: introduction and neuroepithelial tumours. In: Seth Love, David N.Louis, David W.Ellison, eds. Greenfield's Neuropathology. London: Hodder Arnold, (2008). , 2008, 1855-60.

[4] Jones, D. T, Gronych, J, Lichter, P, Witt, O, \& Pfister, S. M. MAPK pathway activation in pilocytic astrocytoma. Cell Mol Life Sci (2011).

[5] Johnson, D. R, Brown, P. D, Galanis, E, \& Hammack, J. E. Pilocytic astrocytoma survival in adults: analysis of the Surveillance, Epidemiology, and End Results Program of the National Cancer Institute. J Neurooncol (2012). , 187-93.

[6] Burger, P. C, \& Scheithauer, B. W. Pilocytic astrocytoma. In: Burger PC, Scheithauer BW, eds. Tumors of Central Nervous System. Washington: The Armed Forces Institute of Pathology, (1994). , 1994, 77-94.

[7] Sandalcioglu, I. E, Gasser, T, Wiedemayer, H, Horsch, S, \& Stolke, D. Favourable outcome after biopsy and decompression of a holocord intramedullary spinal cord astrocytoma in a newborn. Eur J Paediatr Neurol (2002). , 6, 179-82.

[8] Tobias, M. E, Mcgirt, M. J, Chaichana, K. L, et al. Surgical management of long intramedullary spinal cord tumors. Childs Nerv Syst (2008). , 24, 219-23.

[9] Burger, P. C, \& Scheithauer, B. W. Tumors of neuroglia and choroid plexus epithelium- Glioblastoma multiforme. In: P.C.Burger, B.W.Scheithauer, eds. Tumors of the central nervous system. Washington: Armed Forces Institute of Pathology., (1994). , 1994, 25-161.

[10] Paixao, B. A, De Oliveira, R. S, Saggioro, F. P, Neder, L, Chimelli, L. M, \& Machado, H. R. In pursuit of prognostic factors in children with pilocytic astrocytomas. Childs Nerv Syst (2010). , 26, 19-28.

[11] Koeller, K. K, \& Rushing, E. J. From the archives of the AFIP: pilocytic astrocytoma: radiologic-pathologic correlation. Radiographics (2004). , 24, 1693-708.

[12] Matsumoto, K, Akagi, K, Abekura, M, et al. Hypothalamic Pilocytic Astrocytoma Presenting with Intratumoral and Subarachnoid Hemorrhage- Case Report. Neurol Med Chir (Tokyo) (1997). , 849-52. 
[13] Abdollahzadeh, M, Hoffman, H. J, Blazer, S. I, et al. Benign cerebellar astrocytoma in childhood: experience at the Hospital for Sick Children 1980-1992. Childs Nerv Syst (1994). , 10, 380-3.

[14] Fernandez, C, Figarella-branger, D, Girard, N, et al. Pilocytic astrocytomas in children: prognostic factors--a retrospective study of 80 cases. Neurosurgery (2003). , 53, 544-53.

[15] Aldape, K, Simmons, M. L, \& Davis, R. L. Discrepancies in diagnoses of neuroepithelial neoplasms: The San Francisco Bay Area Adult Glioma Study. Cancer (2000). , 2342-9.

[16] Kleihues, P, Burger, P. C, \& Aldape, K. D. et al. Glioblastoma. In: David N.Louis, Hiroko Ohgaki, Otmar D.Wiestler, Webster K.Cavenee, eds. WHO Classification of tumors of the Central Nervous System. Lyon: International Agency for Research on Cancer (IARC), (2007). , 2007, 33-49.

[17] Louis, D. N, Reifenberger, G, Brat, D. J, \& Ellison, D. W. Tumors: introduction and neuroepithelial tumors- Glioblastoma. In: Seth Love, David N.Louis, David W.Ellison, eds. Greenfield's Neuropathology. London: Edward Arnold Publishers, (2008). , 2008, 1821-2000.

[18] Neder, L, Marie, S. K, Carlotti, C. G, et al. Galectin-3 as an immunohistochemical tool to distinguish pilocytic astrocytomas from diffuse astrocytomas, and glioblastomas from anaplastic oligodendrogliomas. Brain Pathol (2004). , 14, 399-405.

[19] Eng, L. F, \& Rubinstein, L. J. Contribution of immunohistochemistry to diagnostic problems of human cerebral tumors. J Histochem Cytochem (1978). , 26, 513-22.

[20] Ikota, H, Kinjo, S, Yokoo, H, \& Nakazato, Y. Systematic immunohistochemical profiling of 378 brain tumors with 37 antibodies using tissue microarray technology. Acta Neuropathol (2006). , 111, 475-82.

[21] Mokhtari, K, Paris, S, Aguirre-cruz, L, et al. Olig2 expression, GFAP, and 1p loss analysis contribute to glioma subclassification. Neuropathol Appl Neurobiol (2005). , 53.

[22] Takei, H, Yogeswaren, S. T, Wong, K. K, et al. Expression of oligodendroglial differentiation markers in pilocytic astrocytomas identifies two clinical subsets and shows a significant correlation with proliferation index and progression free survival. $J \mathrm{Neu}^{-}$ rooncol (2008). , 86, 183-90.

[23] Becker, A. P, Caravina, G, Clara, C, \& Reis, R. M. The Role of Immunohistochemistry in Diagnosis and Prognosis of Glioblastoma Patients. Nova Publishers, (2012).

[24] Schonk, D. M, Kuijpers, H. J, Van Drunen, E, et al. Assignment of the gene(s) involved in the expression of the proliferation-related Ki-67 antigen to human chromosome 10. Hum Genet (1989). , 83, 297-9. 
[25] Gerdes, J, Li, L, Schlueter, C, et al. Immunobiochemical and molecular biologic characterization of the cell proliferation-associated nuclear antigen that is defined by monoclonal antibody Ki-67. Am J Pathol (1991). , 138, 867-73.

[26] Burger, P. C, Shibata, T, \& Kleihues, P. The use of the monoclonal antibody Ki-67 in the identification of proliferating cells: application to surgical neuropathology. Am J Surg Pathol (1986). , 10, 611-7.

[27] Bowers, D. C, Gargan, L, Kapur, P, et al. Study of the MIB-1 labeling index as a predictor of tumor progression in pilocytic astrocytomas in children and adolescents. Clin Oncol (2003). , 2968-73.

[28] Roessler, K, Bertalanffy, A, Jezan, H, et al. Proliferative activity as measured by MIB-1 labeling index and long-term outcome of cerebellar juvenile pilocytic astrocytomas. J Neurooncol (2002). , 141-56.

[29] Neder, L, Colli, B. O, Machado, H. R, \& Carlotti, C. G. Jr., Santos AC, Chimelli L. MIB-1 labeling index in astrocytic tumors--a clinicopathologic study. Clin Neuropathol (2004). , 23, 262-70.

[30] Perillo, N. L, Marcus, M. E, \& Baum, L. G. Galectins: versatile modulators of cell adhesion, cell proliferation, and cell death. J Mol Med (Berl) (1998). , 76, 402-12.

[31] Stillman, B. N, Mischel, P. S, \& Baum, L. G. New roles for galectins in brain tumors-from prognostic markers to therapeutic targets. Brain Pathol (2005). , 15, 124-32.

[32] Park, S. H, Min, H. S, Kim, B, Myung, J, \& Paek, S. H. Galectin-3: a useful biomarker for differential diagnosis of brain tumors. Neuropathology (2008). , 28, 497-506.

[33] Borges, C. B, Bernardes, E. S, Latorraca, E. F, et al. Galectin-3 expression: a useful tool in the differential diagnosis of posterior fossa tumors in children. Childs Nerv Syst (2011). , 27, 253-7.

[34] Shumei SongBaoan Ji, Vijaya Ramachandran et al. Overexpressed Galectin-3 in Pancreatic Cancer Induces Cell Proliferation and Invasion by Binding Ras and Activating Ras Signaling. PLoS ONE (2012). , 7, 1-11.

[35] Huang, H, Hara, A, Homma, T, Yonekawa, Y, \& Ohgaki, H. Altered expression of immune defense genes in pilocytic astrocytomas. J Neuropathol Exp Neurol (2005). , 64, 891-901.

[36] Rorive, S, Maris, C, Debeir, O, et al. Exploring the distinctive biological characteristics of pilocytic and low-grade diffuse astrocytomas using microarray gene expression profiles. J Neuropathol Exp Neurol (2006). , 65, 794-807.

[37] Beatriz, M, Lopes, S, \& Scott, R. VandenBerg. Tumors of the central nervous system. In: Christopher D.M.Fletcher, ed. Diagnostic Histopathology of Tumors. Philadelphia: Elsevier, (2007). , 2007, 1653-732. 
[38] Roberts, P. J. Der CJ. Targeting the Raf-MEK-ERK mitogen-activated protein kinase cascade for the treatment of cancer. Oncogene (2007). , 26, 3291-310.

[39] Dhillon, A. S, Hagan, S, Rath, O, \& Kolch, W. MAP kinase signalling pathways in cancer. Oncogene (2007). , 26, 3279-90.

[40] Marshall, C. J. Ras effectors. Curr Opin Cell Biol (1996). , 8, 197-204.

[41] Dunn, K. L, Espino, P. S, Drobic, B, He, S, \& Davie, J. R. The Ras-MAPK signal transduction pathway, cancer and chromatin remodeling. Biochem Cell Biol (2005). , 83, 1-14.

[42] Yoon, S, \& Seger, R. The extracellular signal-regulated kinase: multiple substrates regulate diverse cellular functions. Growth Factors (2006). , 24, 21-44.

[43] Mckay, M. M, \& Morrison, D. K. Integrating signals from RTKs to ERK/MAPK. Oncogene (2007). , 26, 3113-21.

[44] Vakiani, E, \& Solit, D. B. KRAS and BRAF: drug targets and predictive biomarkers. J Pathol (2011). , 223, 219-29.

[45] Wellbrock, C, Karasarides, M, \& Marais, R. The RAF proteins take centre stage. Nat Rev Mol Cell Biol (2004). , 5, 875-85.

[46] Davies, H, Bignell, G. R, Cox, C, et al. Mutations of the BRAF gene in human cancer. Nature (2002). , 417, 949-54.

[47] Fisher, R, \& Larkin, J. Vemurafenib: a new treatment for BRAF-mutated advanced melanoma. Cancer Manag Res (2012). , 600

[48] Riemenschneider, M. J, Jeuken, J. W, Wesseling, P, \& Reifenberger, G. Molecular diagnostics of gliomas: state of the art. Acta Neuropathol (2010). , 120, 567-84.

[49] Basto, D, Trovisco, V, Lopes, J. M, et al. Mutation analysis of B-RAF gene in human gliomas. Acta Neuropathol (2005). , 109, 207-10.

[50] Schindler, G, Capper, D, Meyer, J, et al. Analysis of BRAF mutation in 1,320 nervous system tumors reveals high mutation frequencies in pleomorphic xanthoastrocytoma, ganglioglioma and extra-cerebellar pilocytic astrocytoma. Acta Neuropathol (2011). , 600E

[51] Bar, E. E, Lin, A, Tihan, T, Burger, P. C, \& Eberhart, C. G. Frequent gains at chromosome 7q34 involving BRAF in pilocytic astrocytoma. J Neuropathol Exp Neurol (2008). , 67, 878-87.

[52] Pfister, S, Janzarik, W. G, Remke, M, et al. BRAF gene duplication constitutes a mechanism of MAPK pathway activation in low-grade astrocytomas. J Clin Invest (2008). , $118,1739-49$. 
[53] Rodriguez, F. J, Ligon, A. H, Horkayne-szakaly, I, et al. BRAF Duplications and MAPK Pathway Activation Are Frequent in Gliomas of the Optic Nerve Proper. J Neuropathol Exp Neurol (2012). , 71, 789-94.

[54] Jentoft, M, Giannini, C, Cen, L, et al. Phenotypic variations in NF1-associated low grade astrocytomas: possible role for increased mTOR activation in a subset. Int $J$ Clin Exp Pathol (2010). , 4, 43-57.

[55] Tian, Y, Rich, B. E, Vena, N, et al. Detection of KIAA1549-BRAF fusion transcripts in formalin-fixed paraffin-embedded pediatric low-grade gliomas. J Mol Diagn (2011). , 13, 669-77.

[56] Jones, D. T, Kocialkowski, S, Liu, L, Pearson, D. M, Ichimura, K, \& Collins, V. P. Oncogenic RAF1 rearrangement and a novel BRAF mutation as alternatives to KIAA1549:BRAF fusion in activating the MAPK pathway in pilocytic astrocytoma. Oncogene (2009). , 28, 2119-23.

[57] Ida, C. M, Lambert, S. R, Rodriguez, F. J, et al. BRAF alterations are frequent in cerebellar low-grade astrocytomas with diffuse growth pattern. J Neuropathol Exp Neurol (2012). , 71, 631-9.

[58] Lin, A, Rodriguez, F. J, Karajannis, M. A, et al. BRAF alterations in primary glial and glioneuronal neoplasms of the central nervous system with identification of 2 novel KIAA1549:BRAF fusion variants. J Neuropathol Exp Neurol (2012). , 71, 66-72.

[59] Yadav, V, Zhang, X, Liu, J, Estrem, S, Li, S, Gong, X-Q, Buchanan, S, Henry, J. R, Starling, J. J, \& Peng, S-B. Reactivation of Mitogen-Activated Protein Kinase (MAPK) Pathway by FGF Receptor 3 (FGFR3)/Ras Mediates Resistance to Vemurafenib in Human B-RAF Mutant Melanoma. Journal of Biological chemistry, 1-20. (2012). Ref Type: In Press, 600E

[60] Gautschi, O, Pauli, C, Strobel, K, Hirschmann, A, Printzen, G, Aebi, S, \& Diebold, J. A patient with BRAF lung adenocarcinoma responding to Vemurafenib. Journal of Thoracic Oncology 28. (2012). Ref Type: In Press, 600E

[61] Flaherty, K. T, Robert, C, Hersey, P, et al. Improved Survival with MEK Inhibition in BRAF-Mutated Melanoma. The New England Journal of Medicine (2012). , 367, 107-14.

[62] Flaherty, K. T, Puzanov, I, Kim, K. B, et al. Inhibition of mutated, activated BRAF in metastatic melanoma. N Engl J Med (2010). , 363, 809-19.

[63] Druker, B. J, Guilhot, F, \& Brien, O. SG et al. Five-year follow-up of patients receiving imatinib for chronic myeloid leukemia. N Engl J Med (2006). , 355, 2408-17. 
